
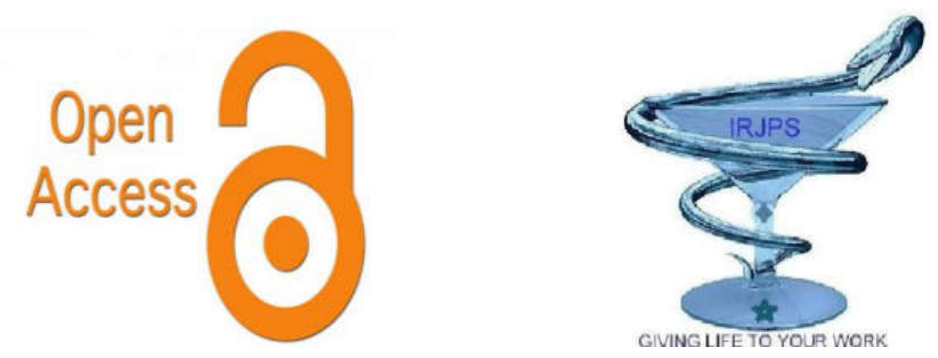

\title{
REVIEW
}

This is on open access article which permits unrestricted non-commercial use, provided it is properly cited. ISSN (0): 2349-5332 CODEN: IRJPHY

\section{SHORT TERM RENAL AFFECTS OF SODIUM-GLUCOSE LINKED TRANSPORTER 2 INHIBITORSIN CHRONIC KIDNEY DISEASES - A REVIEW}

\section{G. D. S. Chaitanya*, Namagiri Manisha.}

Avanthi College of Pharmaceutical Science, Vizianagaram, Andhra Pradesh.

Submitted on: 26.09.18;

Revised on: 30.09.18;

Accepted on: 06.10.18

\begin{abstract}
:
Sodium-glucose linked transporter 2 (SGLT 2) inhibitors are a new of anti-diabetic drugs. In contrast to other antidiabetic drugs, these are insulin independent in action. These drugs provide additional benefits when used in combination with other class of drugs. Along with glucose lowering potential, these drugs exhibit some pleotropic effects. One among them is reduction single nephron glomerular filtration. SGLT 2 inhibitors are found to be effective in patients with normal glomerular filtration rate (GFR). But certain considerations are needed for the use of these drugs in patients with renal impairment. Their effect on renal function and related adverse outcomes were not dealt properly. In this paper, we provide a review regarding effect of SGLT 2 inhibitors on renal function in diabetic patients with renal impairment.
\end{abstract}

KEY WORDS: SGLT 2 inhibitors, Diabetes mellitus, Renal function, Glomerular Filtration Rate.

Corresponding Author: G. D. S. Chaitanya E-Mail: chaitanyacherry007@gmail.com
Indian Research Journal of Pharmacy and Science; 17(2018)1636-1641; Journal Home Page: https://www.irjps.in DOI: 10.21276/irjps.2018.5.3.10 


\section{INTRODUCTION:}

Diabetes is one of the major health problems worldwide and its prevalence has nearly doubled since 1980 rising from $4.7 \%$ to $8.5 \%$ in adult population. ${ }^{1}$ It is a group of metabolic diseases characterized by hyperglycemia resulting from defects in insulin secretion, insulin action, or both. The chronic hyperglycemia of diabetes is associated with long-term damage and failure of various organs, especially the eyes, kidneys, nerves, heart, and blood vessels. It is one of the leading causes of renal diseases. It is estimated that approximately one-third of patients with type 2 diabetes have some degree of renal impairment. ${ }^{2}$

Chronic kidney disease (CKD) is characterized by a permanent damage to nephrons. The loss of renal mass induces compensatory condition of glomerular hyper filtration and tubular hypertrophy which leads to glomerular sclerosis that progress towards end stage renal disease.

The risk of microvascular complications, including retinopathy, nephropathy and neuropathy can be reduced by intensive glycemic control in patients with type 2 diabetes mellitus. ${ }^{3,4}$ In most of the patients with type 2 diabetes mellitus (DM) beta cell function continues to decline as their disease progresses. Medications that rely on insulin secretion are characterized by high rate of secondary failure and are useful only in the initial phases of diabetes, but most of the patients ultimately rely on insulin for glycemic control.

Sodium-glucose linked transporter 2 (SGLT 2) inhibitors, the most recent glucose-lowering oral agents, may have the potential to exert nephroprotective action not only through improving glycemic control but also through glucoseindependent effects, such as blood pressure-lowering and direct renal effects. It is important to consider, however, that in patients with impaired renal function, given their mode of action, SGLT 2 inhibitors are less effective in lowering blood glucose.

\section{PURPOSE OF WORK:}

To assess the safety of SGLT 2 inhibitors use in type
2 diabetes mellitus patients with CKD.

\section{SGLT 2 INHIBITORS:}

Kidney maintains homeostasis by re-absorption of filtered glucose. Two different types of $\mathrm{Na}+/$ glucose transporters involved in handling renal glucose are SGLT1 and SGLT 2. SGLT 2 inhibitors work by inhibiting SGLT 2 in proximal convoluted tubule which is responsible for reabsorption of $80-90 \%$ of glucose. Thus, glucose is excreted in large quantities through urine resulting in fall of plasma glucose levels that helps control glycemic parameters. ${ }^{5-7}$ In contrast to other anti hyperglycemic agents these drugs are insulin independent. The mechanism of action is complementary and not an alternative to mechanism of other anti-diabetic agents. Thus SGLT 2 inhibitors may be suitable for use in a combination of approaches.

Their effects include reduction of hyperglycemia secondary to reduction of $\mathrm{T}_{\mathrm{mG}}$ (maximum renal glucose re-absorptive capacity also called tubular max for glucose) and attendant increase of urinary glucose excretion. Because these drugs cause glucose excretion they may cause weight loss. Changes in weight may contribute to blood pressure lowering effect of SGLT 2 inhibitors. ${ }^{8}$ SGLT 2 Inhibitors also reduce the single nephron glomerular filtration rate which may be determined by various complex changes in tubule-glomerular feedback. Inhibition of sodium re-absorption leads to increased $\mathrm{Na}+$ delivery to macula densa, which stimulates tubule-glomerular feedback and afferent arterial vasoconstriction and reduces glomerular hyperfiltration. ${ }^{9}$

\section{In Chronic kidney disease:}

The magnitude of glucose excretion and $\mathrm{HbAlc}$ reduction induced by SGLT 2 inhibitors is dependent upon filtered glucose load. Glucose excretion is maximal in diabetics with normal GFR and is modest in renal impairment patients who have reduced filtered glucose load. In patients with reduced GFR, the amount of glucose reaching PCT falls and the efficacy of SGLT 2 inhibitors may be suboptimal. ${ }^{10}$ As the mechanism of action depends on glomerulo-tubular function certain considerations need to be taken in patients with renal impairment. 


\section{Effect on renal function:}

Few studies have been conducted to study the efficacy of SGLT 2 inhibitors in type 2 diabetes patients with renal impairment. One among the outcomes of these studies mentioned, that is effect on eGFR (measure of renal function) is considered.

A 104 week study included 1161 patients, among which 482 patients were assigned to glimeperide $1 \mathrm{mg}, 483$ patients to canagliflozin $100 \mathrm{mg}$ and 485patients to canagliflozin $300 \mathrm{mg}$ groups. Baseline GFR is $90 \mathrm{ml} / \mathrm{min}$. The change in eGFR of subjects from baseline is $-5.4 \mathrm{ml} / \mathrm{min}$ per $1.73 \mathrm{~m}^{2}$ in glimeperide group,-2.7 $\mathrm{ml} / \mathrm{min}$ per $1.73 \mathrm{~m}^{2}$ in cangliflozin $100 \mathrm{mg}$ and $-3.9 \mathrm{ml} / \mathrm{min}$ per $1.73 \mathrm{~m}^{2}$ in canagliflozin $300 \mathrm{mg}$. Patients taking canagliflozin had rapid decrease in eGFR levels in first 4 weeks and later trended towards baseline. ${ }^{11}$

A randomized placebo controlled trial having included 269 subjects was conducted to study efficacy of canagliflozin in type $2 \mathrm{DM}$ and CKD stage 3. Decrease in eGFR was observed in all groups and larger in canagliflozin 100mg (-9.1\%) and canagliflozin 300mg (-10.1\%) compared to placebo ($4.5 \%){ }^{12}$

Comparing above mentioned studies reveal that use of canagliflozin worsens the GFR in subjects with moderate renal impairment within few weeks of use.
A pooled analysis of 11 phase 3 trials assessed the changes in HbAlc, body weight, blood pressure, haematocrit and urine albumin-creatinine ratio with placebo or dapagliflozin 10mg. patients were followed for 24 weeks. Dapagliflozin caused an initial decrease in eGFR.GFR was stable in Dapagliflozin group during subsequent follow-up but did not revert to baseline. After 24 weeks, change in eGFR was -1.7 and $-1.4 \mathrm{ml} / \mathrm{min} / 1.73 \mathrm{~m}^{2}$ in moderate and mild renal impairment patients respectively. No remarkable change was found in patients with GFR above $90 \mathrm{ml} / \mathrm{min}$ per $1.73 \mathrm{~m}^{2}{ }^{13}$

A study was conducted on 252 subjects to assess daily treatment with dapagliflozin in inadequately controlled type 2 diabetes mellitus and moderate renal impairment. After 24 weeks, change in eGFR from baseline was -2.5 for dapagliflozin $5 \mathrm{mg}$ and 5.0 for dapagliflozin10mg. No significant change is observed in placebo group. This shows that dapagliflozin had potential to harm the kidney function. GFR in both groups trended towards baseline during follow up but didn't reach it. ${ }^{14}$

Results of EMPA REG-OUTCOME trial showed that empagliflozin reduces GFR in the same way as other two SGLT 2 inhibitors do. It is observed that eGFR is stable during follow up and is better than that of placebo after 102 weeks. ${ }^{15}$

Table 1: Renal effects in studies of SGLT 2 inhibitions on subjects with moderate chronic kidney diseases.

\begin{tabular}{|c|c|c|c|}
\hline Study- 1 & Placebo & Canagliflozin 100mg & Canagliflozin 300mg \\
\hline Baseline GFR $\left(\mathrm{ml} / \mathrm{min} / 1.73 \mathrm{~m}^{2}\right)$ & 40.1 & 39.7 & 38.5 \\
\hline Change in eGFR after 3 weeks & $-4.5 \%$ & $-9.1 \%$ & $-10.1 \%$ \\
\hline Study- 2 & Placebo & Dapagliflozin 5mg & Dapagliflozin 10mg \\
\hline Baseline GFR $\left(\mathrm{ml} / \mathrm{min} / 1.73 \mathrm{~m}^{2}\right)$ & 45.6 & 44.2 & 43.9 \\
\hline Change in eGFR after 3 weeks & -0.25 & -2.38 & -4.80 \\
\hline Study- 3 & Placebo & E mpagliflozin 10mg & E mpagliflozin 25mg \\
\hline Baseline GFR $\left(\mathrm{ml} / \mathrm{min} / 1.73 \mathrm{~m}^{2}\right)$ & 48.6 & 48.4 & 48.4 \\
\hline Change in eGFR after 3 weeks & 0.04 & 2.48 & 3.28 \\
\hline
\end{tabular}

\section{Renal related adverse effects:}

The incidence of adverse effects in clinical trials of SGLT 2 inhibitors is similar to that observed with anti-diabetic drugs. The overall incidence of serious adverse events has ranged from $1.0 \%$ to $12.6 \% .{ }^{16}$ Use of these drugs has been found to increase serum creatinine and blood urea, with a corresponding fall in glomerular filtration rate.

A randomized, active controlled 104 week study of canagliflozin $100 \mathrm{mg}, 300 \mathrm{mg}$ versus glimeperide plus 
metformin was analyzed. Renal related adverse effects like increase in blood creatinine and decrease in eGFR were observed. Incidence of renal related AEs was almost same in three groups. ${ }^{11}$

A randomized, placebo controlled study was conducted to assess treatment with dapagliflozin in 252 patients with type $2 \mathrm{DM}$ and renal impairment. It provided that renal related adverse events were found be occurring at higher rate in patients taking dapagliflozin 5mg. (Dapagliflozin 5mg-9.4\% and Dapagliflozin 10mg-2.4\%). ${ }^{14}$

A study on empagliflozin to assess its efficacy and safety in type $2 \mathrm{DM}$ and renal impairment included 741 patients. Subjects were allotted to empagliflozin 10mg, empagliflozin25mg and placebo groups. Serious adverse events were observed in $6.12 \%$ of empagliflozin $10 \mathrm{mg}$ group and $12.46 \%$ of subjects taking empagliflozin $25 \mathrm{mg}$.Serious renal related adverse effects that include acute and chronic renal failure were observed in $1.55 \%$ of subjects taking empagliflozin $25 \mathrm{mg}$.

The EMPA-REG OUTCOME trials provided that renal related adverse events were reported in a lower percentage in empagliflozin treated group when compared to placebo. ${ }^{15}$

\section{ANALYSIS OF FAERS REPORTS:}

An analysis of AE reports regarding SGLT 2 inhibitors that are submitted to FAERS between Jan 2013 and Sept 2016 was done. Results showed that proportion of reports with ARF among SGLT 2 inhibitors was almost three fold compared to reports without these drugs. Canagliflozin was found to be associated with a high proportion of renal failure $(7.3 \%)$ when compared to dapagliflozin $(4.8 \%)$ and empagliflozin $(4.7 \%){ }^{17}$

Among the reports reviewed by the FDA, around half of the patients developed acute kidney injury within a month of starting an SGLT 2 inhibitor. Most of the cases improved after taking off those drugs from therapy.

Above mentioned studies provide an association between SGLT 2 inhibitors effect on GFR and renal related adverse events. The early onset of acute kidney injury events with SGLT 2 inhibitors probably reflects the acute changes in eGFR attributable to known renal haemo-dynamic effects of SGLT 2 inhibition. This effect seems to be more pronounced in patients having renal impairment.

\section{DISCUSSION:}

Most of previous studies regarding SGLT 2 inhibitors have mentioned their reno-protective effects leaving their short term negative profile in the backyard. SGLT 2 inhibitors are reno-protective on a long term usage. But these drugs are associated with worsening of GFR and renal related adverse outcomes in type 2 DM subjects with renal impairment.

The use of SGLT 2 inhibitors in CKD increases the risk of side effects and should be carefully considered. The use of canagliflozin in patients with moderate CKD is associated with a higher occurrence of renal-related adverse reactions and decreases in GFR compared to patients with mild renal impairment or normal renal function. The use of dapagliflozin in patients with moderate CKD exhibited rapid decrease in GFR and had more renalrelated adverse reactions than placebo-treated patients. Empagliflozin decreases GFR in CKD patients (GFR 30-90 ml/min/1.73 m2) within first few weeks. The efficacy and safety of empagliflozin have not been established in patients with severe renal impairment (GFR $<30 \mathrm{ml} / \mathrm{min} / 1.73 \mathrm{~m} 2)$. Empagliflozin possess less risk of adverse events when compared to other drugs of this class.

\section{CONCLUSION:}

SGLT 2 inhibitors are less effective and also they adversely affect the patients with CKD particularly in case of moderate to severe impairment. Even though the renal function becomes stable on long term usage of these drugs, the short term effects that are worsening the renal function need to be considered. Use of these drugs is associated with high risk of acute renal failure than compared to other classes of anti-hyperglycemic agents. The safety of SGLT 2 inhibitors is still not established in patients with moderate to severe renal impairment and further studies are needed. 


\section{REFERENCES:}

1. WHO: Global report on Diabetes. www.WHO.int

2. Coll-De-Teuro, M. Mata-Cases, A.Rodriguez-Poncelas., Chronic kidney disease in the type 2 diabetic patients: prevalence and associated variables in a random sample of 2642 patients of a Mediterranean area, BMC Nephrology, vol. 13 , no. 1 , article $87,2012$.

3. UKPDS 34, Effect of intensive blood glucose control with metformin on complications in overweight patients with type 2 diabetes (UKPDS). UK Prospective Diabetes Study (UKPDS) Group.The Lancet, vol.352,pp. 854-865,1998.

4. Intensive blood-glucose control with sulphonylureas or insulin compared with conventional treatment and risk of complications in patients with type 2 diabetes

(UKPDS 33). UK prospective diabetes study (UKPDS) Group,” The Lancet, vol. 352, no. 9131, pp. 352-837,1998.

5. Wright EM. Renal $\mathrm{Na}+$ - glucose cotransporters. Am J Physiol Renal Physiol. 2001; 280(1):F10-F18.

6. Lee YJ, Han HJ. Regulatory mechanisms of $\mathrm{Na}+$ /glucose cotransporters in renal proximal tubule cells.Kidney Int suppl. 2007; (106): S27-35.

7. Hummel CS, Lu C, Loo DD Hirayamma BA, Voss AA, Wright EM., Glucose transport by human renal $\mathrm{Na}+/ \mathrm{D}$-glucose transporters SGLT1 and SGLT 2. Am J Physiol Cell Physiol. 2011; 300: C721.

8. S.Engeli, J.Bohnke, K. Gorzelniak.,Weight loss and the renin angiotension-aldosterone system, Hypertension, vol. 45, no. 3, pp. 356-362, 2005.

9. Vallon V, Blantz RC, Thomson S., Glomerular hyperfiltration and the salt paradox in early type 1 diabetes mellitus: a tubule-centric view. J Am Soc Nephrol. 2003; 14: 530-7.

10. L.Zanoli, A. Granata, P.lentini, S. Rastelli, P. Fatuzzo, F.Rapisarda, P.Castellino.,
Sodium-glucose linked transporter-2 inhibitors in chronic kidney disease, The scientific world journal., Vol 2015, Article 317507.

11. Hiddo J. L. Heerspink, Mehul desai, Meg jardine, Dainius balis, Gary meininger, Vlado perkovic, Canagliflozin slows progression of renal function decline independently of glycemic effects, J Am Soc Nephrol., 2017 Jan; 28(1) 368-375.

12. J-F Yale, G Bakris, B Cariou, D Yue, E David neto, L Xi, Efficacy and safety of canagliflozin in subjects with type 2 diabetes and chronic kidney disease, Diabetes Obes Metab., 2013 May; 15(5): 463-473.

13. Sergei Petrykiv, C. David sjostrom, Peter J. Greasley, John $\mathrm{Xu}$, Frederik person, and Hiddo J.L. Heerspink., Differential effects of Dapagliflozin on cardiovascular risk factors at varying degrees of renal function, Clin J Am Soc Nephrol. 2017 May 8; 12(5): 751-759.

14. D.E.Kohan, P.Fioretto, W.Tang, and J.F.Liat., Long-term study of patients with type 2 Diabetes and moderate renal impairment shows that dapagliflozin reduces weight and blood pressure but does not improve glycemic control, kidney international, 2014, Vol. 85, no.4, 962-971.

15. Christoph Wanner, M.D., Silvio E. Inzucchi, M.D., John M. Lachin, Sc.D., David Fitchett, M.D., Empagliflozin and progression of kidney disease in type 2 diabetes. N Engl J Med, July 2016; 375: 323-334.

16. Rossenwasser RF, Sultan S, Sutton D, Choski R, Epstein BJ. SGLT-2 inhibitors and their potential in the treatment of diabetes. Diabetes Metab Syndr Obes. 2013; 6: 453-467.

17. A.Perlman, S.N.Heyman, I. Matok, J.Stokar, M. Muszkat, A.Szalat, Acute renal failure with sodium glucose-co transporter-2 inhibitors: Analysis of the FDA adverse event report system database. NMCD journal. October 17,2017. 


\section{ABBREVIATIONS:}

SGLT 2- Sodium-glucose linked transporter 2

GFR- Glomerular filteration rate

CKD- Chronic kidney disease
DM- Diabetes mellitus

FAERS- FDA adverse event reporting system

eGFR- Estimated glomerular filtration rate 